\title{
Are information provision interventions effective for people with multiple sclerosis? A Cochrane Review summary with
} commentary

\author{
Chiara Arienti \\ IRCCS Fondazione Don Carlo Gnocchi, Via Capecelatro, 66 - 20148 Milan, Italy \\ Tel.: +39 0307245282; E-mail: carienti@dongnocchi.it.
}

\begin{abstract}
.
BACKGROUND: Multiple sclerosis (MS) is one of the major causes of chronic neurologic disability in young adults.

OBJECTIVE: To evaluate the effectiveness of information provision interventions for people with MS that aim to promote informed choice and improve patient-relevant outcomes.

METHODS: To summarize and to discuss the rehabilitation perspective on the published Cochrane Review "Information provision for people with multiple sclerosis" by Köpke $S$ et al.

RESULTS: 11 studies (with 1387 participants) with important clinical heterogeneity on the definitions of interventions and outcomes assessed were included, showing that information provision interventions vs optimized standard care probably slightly increase patients' knowledge and may make little or no difference to decision making and quality of life.

CONCLUSIONS: It is very important to provide information to patients for informed consent and to increase their knowledge and decision-making capacity.
\end{abstract}

Keywords: Multiple sclerosis, Cochrane Systematic Review, information provision interventions, informed choice

The aim of this commentary is to discuss in a rehabilitation perspective the recently published Cochrane Review "Information provision for people with multiple sclerosis" by Köpke S, Solari A, Rahn A, Khan F, Heesen C, Giordano A ${ }^{1}$, under the direct supervision of Cochrane Multiple Sclerosis and Rare Diseases of the CNS Group. This Cochrane Corner is produced in agreement with NeuroRehabilitation by Cochrane Rehabilitation.

\footnotetext{
${ }^{1}$ The abstract/plain language summary of this Cochrane Review is taken from a Cochrane Review previously published in the Cochrane Database of Systematic Reviews 2018, Issue
}

\section{Background}

Multiple sclerosis (MS) is an autoimmune inflammatory demyelinating disease of the central nervous system that affects approximately 1.3 million people

10, art. no. CD008757. DOI: http://dx.doi.org/10.1002/14651858. CD008757.pub3 (see www.cochranelibrary.com for information). Cochrane Reviews are regularly updated as new evidence emerges and in response to feedback, and Cochrane Database of Systematic Reviews should be consulted for the most recent version of the review. The views expressed in the summary with commentary are those of the Cochrane Corner author and do not represent the Cochrane Library or Wiley. 
worldwide. It is one of the major causes of chronic neurologic disability in young adults, associated with complexity that lead to progressive limitation of functioning in daily life, requiring long-term multidisciplinary management (Khan \& Amatya, 2017).

In this complex context, it is very important for these patients to have access to adequate information in order to make informed choices to evaluate the effectiveness of different treatment options (Saini et al., 2017).

The significant question addressed in this review of how information provision interventions can promote informed choice and improve patient-relevant outcomes would be of great importance for rehabilitation professionals that often have to manage the communication with patients and their caregivers.

Information provision for people with multiple sclerosis

(Köpke S, Solari A, Rahn A, Khan F, Heesen C, Giordano A, 2018)

\section{Objective}

The primary aim of this Cochrane Review was to evaluate the effectiveness of information provision interventions for people with multiple sclerosis (MS) that aim to promote informed choice and improve patient-relevant outcomes.

\section{What was studied and methods}

The studies included were randomised, clusterrandomised, or quasi-randomised trials of information provision interventions provided with the idea of improving patient-relevant outcomes compared with standard care; and trials comparing two different strategies of information provision were included. The population included was patients of all ages with a diagnosis of any course of MS or in the diagnostic process. The interventions studied were any intervention or group of interventions providing disease-specific information given with the aim of improving patient-relevant outcomes. The intervention was compared to standard care or optimized standard care. The outcomes studied as primary were: disease-related ('risk') knowledge and measures of (shared) decision making. The secondary measures were: quality of life, measures of informed choice, psychological status measures, treatment choices and adherence, satisfaction with the information received and with the decisional process, hospital admissions and use of health services, measures of activities of daily living, coping, disability, role preferences and adverse events.

\section{Results}

The review included 11 studies (with 1387 participants) with important clinical heterogeneity on the definitions of interventions and outcomes assessed. The review did not evaluate statistical heterogeneity and did not perform meta-analyses. The review presented the results in a narrative format and showed that information provision interventions vs optimized standard care probably slightly increase patients' knowledge (moderate-certainty evidence) and may make little or no difference to decision making and quality of life (low-certainty evidence). No adverse events were found.

\section{Conclusions}

The authors concluded that information provision for people with MS probably increases diseaserelated knowledge, with less clear results on decision making and quality of life. The included studies reported no negative side effects of providing diseaserelated information to people with MS. Interpretation of study results remains challenging due to the marked heterogeneity of interventions and outcome measures.

\section{Implications for practice in neurorehabilitation}

This review evaluated the effectiveness of information provision interventions for people with MS that aim to promote informed choice and improve patient-relevant outcomes.

The results show that all interventions included information provision as at least one of the main intervention components, but the main goals were different between the studies. Therefore, this heterogeneity does not allow clear clinical suggestions, but highlights that information provision interventions probably increase disease-related knowledge and may have a positive impact on decision making and quality of life. 
MS is a complex health condition with various disabilities impacting function and quality of life. From the rehabilitation perspective, it is very important to provide information to patients for informed consent and to increase their knowledge and decision-making capacity.

\section{Acknowledgments}

The author thanks Cochrane Rehabilitation and Cochrane Multiple Sclerosis and Rare Diseases of the CNS Group for reviewing the contents of the Cochrane Corner.

\section{Conflict of interest}

The author declares no conflicts of interest.

\section{References}

Khan, F., \& Amatya, B. (2017). Rehabilitation in Multiple Sclerosis: A Systematic Review of Systematic Reviews. Archives of Physical Medicine and Rehabilitation, 98(2), 353-367. https://doi.org/10.1016/j.apmr.2016.04.016

Köpke, S., Solari, A., Rahn, A., Khan, F., Heesen, C., \& Giordano, A. (2018). Information provision for people with multiple sclerosis. The Cochrane Database of Systematic Reviews, 10, CD008757. http://dx.doi.org/10.1002/ 14651858.CD008757.pub3

Saini, V., Garcia-Armesto, S., Klemperer, D., Paris, V., Elshaug, A. G., Brownlee, S., . . Fisher, E. S. (2017). Drivers of poor medical care. Lancet (London, England), 390(10090), 178-190. https://doi.org/10.1016/S0140-6736(16)30947-3 